\title{
Síndromes de disfunción autonómica asociados con intolerancia ortostática
}

\author{
Blair P. Grubb ${ }^{1}$, Boris E. Vesga ${ }^{2,3}$, Juan Camilo Guzmán ${ }^{2}$, \\ Federico A. Silva ${ }^{2,4}$, Carlos A. Morillo ${ }^{2,4,5}$ \\ ${ }^{1}$ Medical College of Ohio, Toledo, Ohio, U.S.A. \\ 2 Instituto de Investigaciones Fundación Cardiovascular del Oriente Colombiano, Bucaramanga, Colombia. \\ 3 Universidad Autónoma de Bucaramanga, Bucaramanga, Colombia. \\ ${ }^{4}$ Departamento de Medicina Interna, Universidad Industrial de Santander, Bucaramanga, Colombia. \\ ${ }^{5}$ Departamento de Medicina, McMaster University, Hamilton, Ontario, Canadá.
}

Los síndromes de disfunción autonómica asociados con la intolerancia ortostática son una de las alteraciones cuya fisiopatología fue reconocida hace sólo pocos años, lo que ha llevado a un mejor reconocimiento clínico. Entre las manifestaciones clínicas, el síncope y el presíncope asociados con ortostatismo son muy frecuentes. El espectro de la presentación clínica de estos síndromes varía desde la fatiga crónica hasta el síncope neurocardiogénico recurrente. En este artículo se revisa la fisiopatología y clasificación actual de los síndromes de disfunción autonómica asociados a intolerancia ortostática, así como las causas de disautonomía primaria y secundaria, y su enfoque diagnóstico y terapéutico.

Palabras clave: intolerancia ortostática, disfunción autonómica, función autonómica, síncope mediado neuralmente, síncope vasovagal.

\begin{abstract}
Recent advances in diagnosis of autonomic disfunction syndromes associated with orthostatic intolerance

In recent years increased interest has focused on the nature and pathophysiology of orthostatic intolerance and syndromes associated with autonomic disorders. Understanding the pathophysiology underlying these syndromes has led to the recognition of several distinct clinical entities with overlapping features and the associated need to reclassify many of the previously unrecognized syndromes. Among the clinical manifestations, syncope and near syncope are frequently associated with orthostatic intolerance. In addition, however, a wide spectrum of symptoms have been described ranging from chronic fatigue to recurrent neurally mediated vasodepressor reactions. The present review focuses on the pathophysiology and classification of syndromes of autonomic dysfunction associated with orthostatic intolerance. Primary and secondary causes of dysautonomia as well as therapeutic approach to these frequently unrecognized syndromes is presented.
\end{abstract}

Key words: orthostatic intolerance, autonomic failure, autonomic function, neurally mediated syncope, vasovagal syncope.

El sistema nervioso autónomo (SNA) como eje central del control cardiovascular modula los reflejos relacionados con el control de la presión

Correspondencia:

Carlos A. Morillo, Cardiology División-Arrhythmia Service, McMaster Clinic, 5th Floor, Room 503, 237 Barton Street East Hamilton, Ontario, Canada L8L 2X2

Phone: (905) 577-8004, Fax: (905) 521-8820

morillo@hhsc.ca

Recibido: 2/0802; aceptado: 17/01/03 arterial y la frecuencia cardíaca, lo que permite mantener un gasto cardíaco adecuado en respuesta al ortostatismo. El estudio fisiopatológico y la caracterización clínica de los síndromes de disfunción autonómica que se manifiestan con intoleracia ortostática han sido un reto para los investigadores de las áreas básicas y clínicas debido a su frecuente presentación y a la falta de herramientas terapéuticas que permitan su adecuado manejo. 
Teniendo en cuenta que son el médico general y el internista quienes inicialmente examinan al paciente con intolerancia ortostática y síncope y que con frecuencia éste es remitido al cardiólogo y al neurólogo, consideramos pertinente esta revisión actualizada de un problema frecuente en la práctica médica diaria.

A continuación se presenta una revisión descriptiva sobre los síndromes de disfunción autonómica asociados con intolerancia ortostática con base en la información obtenida a partir de la búsqueda en sistemas de referencia como Medline, Proquest y Ovid, complementado con datos provenientes de nuestra casuística.

\section{Sistema nervioso autónomo}

El sistema nervioso humano tiene dos componentes básicos, separados anatómicamente, que trabajan interconectados: el sistema nervioso central (SNC), formado por el cerebro y la médula espinal, y el sistema nervioso periférico, conformado por un grupo de neuronas conectadas a los ganglios y a los nervios que se encuentran fuera del sistema nervioso central (1). El sistema nervioso periférico se subdivide en dos porciones: somática y autonómica. La división somática maneja la información sensitiva de las condiciones extracorporales y la posición muscular y corporal. La división autonómica, conocida como el sistema nervioso autónomo (SNA), controla vísceras, músculo liso corporal, especialmente los vasculares, y glándulas exocrinas. El SNA está compuesto por tres sistemas: simpático, parasimpático y entérico. El sistema simpático ayuda a controlar la reacción del cuerpo ante situaciones de estrés, mientras que el parasimpático trabaja para conservar los recursos corporales y restaurar el equilibrio en estado de reposo. Finalmente, el sistema entérico es el encargado de regular y controlar la función intestinal (2).

Los sistemas corporales gobernados por el SNA son independientes del control voluntario, pero pueden ser influenciados por la voluntad o las emociones. Entre estos sistemas se incluyen el cardiovascular, el respiratorio, el digestivo y el genitourinario. El SNA es vital para el mantenimiento de la homeostasis interna, utilizando para este fin los mecanismos de regulación de la presión sanguínea, fluidos, equilibrio electrolítico y la temperatura corporal (3).

Aún cuando uno de los hechos que marcaron la evolución del homo sapiens fue la adopción de la postura erguida y, por ende, la bipedestación, esta situación fue un desafío para el sistema de control de la presión sanguínea, lo que resultó en que el cerebro tenga una ubicación precaria con respecto a la perfusión vascular y la oxigenación.

EI SNA es el que gobierna las respuestas de la presión sanguínea al cambio posicional a corto y mediano plazo (4). Normalmente, el $25 \%$ del volumen de la sangre circulante está en el tórax; una vez se toma la posición erecta, la gravedad produce un brusco desplazamiento descendente de $500 \mathrm{ml}$ de sangre hacia el abdomen y los miembros inferiores; aproximadamente el $50 \%$ de este volumen se redistribuye en los siguientes segundos. Ello causa una disminución en el retorno venoso al corazón y en las presiones de llenado cardíaco, lo cual puede llevar a disminuir el volumen de eyección en un $40 \%$ (5). El punto de referencia para la determinación de estos cambios se conoce como el punto de indiferencia hidrostática $(\mathrm{PIH})$ venosa, que representa la parte del sistema vascular donde la presión es independiente de los cambios posturales. En los humanos, la PIH está ubicada al nivel del diafragma, mientras que la PIH arterial se encuentra al nivel del ventrículo izquierdo. La PIH venosa es dinámica, debido a que puede ser alterada por cambios en la capacitancia venosa causados por la actividad muscular (4).

En un sujeto normal, la estabilización ortostática se obtiene en un minuto o menos después de ponerse de pie. Se debe tener en cuenta que las respuestas circulatorias exactas aparecen en la posición erecta (proceso activo) y son algo diferentes a las inducidas en la prueba de la mesa basculante al tomar la posición erguida de manera pasiva; en los momentos siguientes a la adopción de la postura erguida ocurre un descenso lento en la presión arterial y en el llenado cardíaco; esto causa la activación de los mecanorreceptores de alta presión localizados en el seno carotídeo y el arco aórtico, así como los de baja presión ubicados en el corazón y los pulmones. 
Los mecanorreceptores ubicados dentro de las aurículas y ventrículos están unidos a través de fibras vagales aferentes no mielínicas $(1,3,4,6,7)$, las cuales han sido señaladas como el origen de las señales inhibitorias que suceden de forma continua sobre las áreas cardiovasculares a nivel medular y del núcleo del tracto solitario (1). La caída en el retorno venoso, que sucede al tomar la postura erguida, produce un menor estiramiento de estos receptores, disminuyendo la descarga aferente al sistema nervioso y causando un incremento en la descarga simpática, lo que genera vasoconstricción sistémica. De forma simultánea, la caída de la presión arterial en la posición erguida estimula los receptores de alta presión ubicados en el seno carotídeo, reduciendo la descarga vagal eferente al nodo sinusal y produciendo un incremento de la frecuencia cardiaca. Las adaptaciones tempranas al tomar la posición erguida dan como resultado un incremento de la frecuencia cardíaca de 10 a 15 latidos por minuto y de la presión diastólica de $10 \mathrm{~mm} \mathrm{Hg}$, con un cambio mínimo o nulo en la presión sistólica. Una vez los ajustes se han completado, si se compara la posición de pie con la posición supina, el volumen sanguíneo torácico es un $30 \%$ menor, así como el gasto cardíaco; de forma adicional existe un aumento de la frecuencia cardiaca de 10 a 15 latidos/minuto. Si la persona continúa de pie, se presenta activación de las respuestas neurohumorales de acuerdo con el volumen corporal. Como regla, cuanto más bajo sea el volumen, mayor será el grado de activación del sistema renina-angiotensina-aldosterona.

La incapacidad de cualquiera de estos procesos de funcionar adecuadamente o de forma coordinada, puede resultar en una falla en la respuesta normal a cambios posturales súbitos o en el mantenimiento postural, lo cual genera hipotensión, elementos que pueden ser suficientes para producir hipoperfusión cerebral, hipoxia y pérdida de la conciencia.

\section{Desórdenes del control ortostático}

Recientemente se ha identificado un buen número de alteraciones del control ortostático que, aunque comparten ciertas características, son entidades únicas. Con el fin de poner orden al aparente caos en el sistema de clasificación de estos desórdenes, generado por la aparición de múltiples clasificaciones, algunas arbitrarias y que han generado grandes discusiones, la Sociedad Americana del Sistema Nervioso Autónomo creó en 1996 (8) una clasificación con el fin de unificar criterios. La disfunción autonómica asociada con intolerancia ortostática se puede subdividir en: 1) síncope reflejo: síncope cardiogénico, hipersensibilidad del seno carotídeo y misceláneo (micción, posprandial, defecación); 2) síndrome de taquicardia ortostática postural con taquicardia sinusal inapropiada: hipersensibilidad betaadrenérgica, intolerancia ortostática, aumento del automatismo del nodo sinusal; 3) falla autonómica pura: aguda y crónica (primaria o secundaria)], y 4) atrofia sistémica múltiple: parkinsoniana, cerebelar y mixta. Varios investigadores prefieren dividir este tipo de desórdenes en formas primarias y secundarias. Las formas primarias tienden a ser idiopáticas y están subdivididas en formas agudas y crónicas. Las formas secundarias frecuente-mente se asocian con enfermedades específicas o secundarias a anormalidades bioquímicas.

\section{Síncope reflejo}

El síncope se define como la pérdida transitoria del estado de conciencia y el tono postural. Es una de las enfermedades humanas más tempranamente descritas: Hipócrates realizó su primera descripción y el origen de la palabra proviene del griego syncoptein (cortar abruptamente), de donde se deriva el término para denominar el desmayo. El síncope tiene como causa una gran cantidad de etiologías que incluyen un amplio espectro de enfermedades cardiacas, metabólicas y neurológicas. En los últimos tres lustros se ha centrado mucha atención en una de las causas más frecuentes de pérdida del conocimiento, el síncope vasovagal. Las investigaciones dirigidas a identificar la naturaleza de este desorden han demostrado que éste es sólo una de las causas de un amplio grupo de alteraciones del sistema nervioso autónomo que pueden llevar a hipotensión, intolerancia ortostática y, finalmente, al síncope.

El síncope reflejo, descrito simultáneamente por Gower y Sir Thomas Lewis como síncope vasovagal, es mejor conocido hoy como síncope 
neurocardiogénico o síncope mediado neuralmente. Aunque las características de su presentación son variadas, se presenta con mayor frecuencia en sujetos jóvenes y se caracteriza por un pródromo típico de duración variable, seguido de una pérdida abrupta de la conciencia. La recuperación es rápida y usualmente no se acompaña de un estado postictal.

Estos episodios sincopales sugieren la presencia de una hipersensibilidad del SNA que responde de forma exagerada a varios estímulos, entre los cuales se destaca el estrés ortostático prolongado. Al asumir la posición erecta se induce un incremento en la capacitancia venosa, que reduce el retorno venoso al ventrículo derecho, lo que precipita un incremento inotrópico que finalmente lleva a la activación de los mecanorreceptores, los cuales normalmente se disparan durante el estiramiento (6). Este aumento súbito en el tráfico neural hacia el tallo cerebral simula las condiciones observadas en la hipertensión arterial, lo que provoca un retiro abrupto de la actividad simpática que lleva a hipotensión, bradicardia y síncope.

En estudios en los que se ha registrado la actividad simpática neural muscular, mediante microneurografía del nervio peroneal, se documentó una supresión abrupta del tráfico simpático previo al inicio de la hipotensión y la bradicardia $(9,10)$. Igualmente, se ha documentado una disminución en la ganancia barorrefleja en sujetos con síncope neurocardiogénico reproducido por la mesa basculante. Existen otros estímulos, como las emociones fuertes o las descargas epilépticas, que pueden provocar una respuesta similar, lo cual hace suponer que estos sujetos tienen un incremento inherente en la sensibilidad a esos estímulos. Durante la estimulación ortostática, lograda mediante la prueba de la mesa basculante, estas personas tienen una caída súbita y profunda en la presión arterial, que se correlaciona con la disminución de la frecuencia cardíaca, en ocasiones, hasta el punto de asistolia (11).

Sutton inicialmente observó que la respuesta presentada durante el síncope neurocardiogénico y la hipersensibilidad del seno carotídeo eran similares y postuló que pueden ser diferentes facetas de un mismo desorden (12). Morillo y colaboradores aportaron algunos elementos suficientes para probar esta hipótesis al documentar la presencia de una reducción en la ganancia barorrefleja basal en pacientes con hipersensibilidad del seno carotídeo asociado con respuestas vasovagales e intolerancia ortostática (13). Adicionalmente, en personas predispuestas, la activación rápida de los mecanorreceptores ubicados en otros lugares, como la vejiga y el tracto respiratorio, puede provocar respuestas similares (14). Lo que parece distinguir estos desórdenes de los demás que se presentan en este artículo, es que estos sujetos son completamente normales entre episodios. Es importante tener en cuenta que, en el caso anterior, el SNA funciona normalmente, a pesar de presentar una respuesta hipersensible ante diversos estímulos, en contraposición con otras patologías en las que el SNA parece tener alteraciones basales.

\section{Disfunción autonómica primaria}

\section{Desórdenes crónicos}

La manifestación clínica de disfunción autonómica crónica es mucho más frecuente que la presentación aguda de estos desórdenes. Bradbury y Eggleston, en 1925, realizaron el primer informe de la disfunción autonómica crónica, patología que fue denominada 'hipotensión ortostática idiopática', debido a la aparente ausencia de otros hallazgos neurológicos (15). Sin embargo, desde entonces es claro que en estos pacientes existe un estado generalizado de disfunción autonómica que clínicamente se manifiesta con hipotensión ortostática y síncope, así como con alteraciones en la función del tubo digestivo, la vejiga, la termorregulación, sudomotora y sexual. La actual clasificación de la Sociedad Autonómica Americana ha clasificado esta entidad como falla autonómica pura (FAP) (16). Dado que la causa de la FAP se desconoce, varios investigadores han postulado la existencia de una degeneración de las neuronas autonómicas periféricas a nivel postganglionar. Aunque la mayoría de los sujetos identificados son adultos mayores, esta alteración puede ocurrir en cualquier etapa de la vida, inclusive en la infancia. 
En 1960, en un artículo ya clásico, Shy y Drager informaron sobre otro tipo de falla autonómica (17). En comparación con la falla autonómica, esta condición se manifiesta por hipotensión ortostática severa, incontinencia fecal y urinaria progresiva, pérdida de la sudoración, atrofia del iris, parálisis de los músculos extraoculares, impotencia, rigidez y temblor; en etapas tardías pueden existir fasciculaciones musculares y atrofia de la musculatura distal. Con el fin de identificar mejor este complejo desorden multisistémico, la Sociedad Autonómica Americana la ha clasificado como atrofia sistémica múltiple (ASM) y la ha dividido en tres subtipos mayores (18): el primer grupo presenta temblor similar al de la enfermedad de Parkinson (degeneración nigroestratial); el segundo grupo presenta síntomas cerebelares o piramidales (atrofia/degeneración olivopontocerebelar), y el tercer grupo presenta características de los dos anteriores. La similitud clínica de la ASM y la enfermedad de Parkinson fue corroborada en un estudio de revisión de autopsias, en el que se encontró que entre el $7 \%$ y el $22 \%$ de los sujetos a los cuales se les había sospechado la enfermedad de Parkinson presentaban elementos neuropatológicos compatibles con ASM. Con respecto a la edad de presentación, la gran mayoría de casos están entre la quinta y la séptima década de la vida, pero, infortunadamente, en algunos sujetos puede iniciar hacia el final de la tercera década de la vida.

Recientemente, se ha generado un gran interés por las formas leves de la falla autonómica crónica. El denominado síndrome de taquicardia ortostática postural (STOP, POTS en inglés) se caracteriza primordialmente por la presencia de taquicardia persistente al asumir la posición erecta que, en ocasiones, alcanza frecuencias de 160 por minuto o más (19). Se asocia, además, a síntomas inespecíficos como fatiga severa, intolerancia al ejercicio y mareos $y$, con frecuencia, se manifiesta con presíncope o síncope recurrente. La mayoría de los pacientes manifiestan sólo síntomas de frialdad, concomitantemente con intolerancia al calor extremo. Durante el reto ortostático mediante la prueba de mesa basculante, se observa un incremento súbito en la frecuencia cardíaca, de más de 30 latidos por minuto durante los primeros cinco minutos, $y$, en ocasiones, se alcanza una frecuencia cardiaca de 120 lpm o más con una leve reducción de la presión arterial.

El mecanismo del STOP parece estar mediado por una disfunción de la circulación periférica para lograr una vasoconstricción adecuada ante el estrés ortostático. Esta deficiencia periférica se compensa mediante un incremento abrupto y excesivo de la frecuencia cardiaca. Varios investigadores han sugerido que el STOP representa un signo temprano de disfunción autonómica y algunos casos tienen una progresión tardía hacia una falla autonómica pura. EI diagnóstico de STOP se establece con base en las características clínicas descritas y la respuesta de la frecuencia cardiaca ante el reto ortostático. Existen dos presentaciones con manifestaciones clínicas diversas. La mayoría de los pacientes tienen una forma leve de disautonomía parcial idiopática, caracterizada por una inhabilidad para aumentar la resistencia vascular periférica ante el reto ortostático, asociada con un aumento exagerado de la frecuencia cardiaca. Clínicamente, estos pacientes presentan una coloración azulada de las extremidades inferiores al ser sometidos a periodos prolongados de bipedestación.

Por otra parte, existe un grupo de pacientes en los cuales la hipersensibilidad beta-adrenérgica es el componente principal. Estos pacientes usualmente son ansiosos y refieren palpitaciones y temblor fino excesivo asociado con la taquicardia postural. Se han documentado, además, respuestas exageradas al reto con isoproterenol y norepinefrina sérica $>600 \mathrm{ng} / \mathrm{ml}$. En estos pacientes, el diagnóstico diferencial con la taquicardia sinusal inapropiada (TSI) es difícil y se basa en las características de las respuestas a las pruebas de función autonómica, especialmente la presencia de un aumento del automatismo del nodo sinusal, que es diagnóstico de la TSI y que no ha sido descrito en los pacientes con STOP (20). Esta diferenciación es importante debido a la posibilidad de establecer diagnósticos equivocados como el de TSI, situación en la cual 
pueden terminar en modificación del nodo sinusal bajo radiofrecuencia y que, después de una aparente eliminación exitosa de la taquicardia, persista la intolerancia ortostática severa. No es claro si estos pacientes presentan una enfermedad distinta o si la TSI es parte del espectro de la disautonomía temprana que lleva al STOP. Otro diagnóstico que puede establecerse de forma errada es el de síndrome de fatiga crónica, situación respecto a la cual varias publicaciones sugieren la posibilidad de una superposición entre estos dos desórdenes $(21,22)$.

\section{Disfunción autonómica aguda}

Las neuropatías autonómicas agudas que producen hipotensión y síncope, se presentan en forma dramática aunque no son comunes (23). En su mayoría, estos desórdenes demuestran un compromiso amplio y severo del sistema simpático y parasimpático, mientras que las fibras somáticas no se ven comprometidas. Muchos casos han sido documentados en jóvenes que previamente eran sanos. El desarrollo de la enfermedad es muy rápido, hasta el punto en el cual los pacientes pueden referir con exactitud el día del inicio de los síntomas. En un porcentaje alto de los casos, se ha observado en estos pacientes una enfermedad febril, presumiblemente viral, previa al inicio de los síntomas, lo cual sugiere la posibilidad de un componente autoinmune agregado.

La función del SNA simpático se encuentra seriamente alterada y la hipotensión ortostática es de tal grado, que el paciente no tolera sentarse en la cama sin presentar síncope. En ocasiones la capacidad de sudar y las funciones intestinal y vesical también están alteradas, siendo una queja frecuente la distensión, las náuseas, el vómito y el dolor abdominal. La constipación es frecuente $y$, en ocasiones, se alterna con diarrea. La frecuencia cardiaca no varía y se encuentra en un rango entre 40 a $50 \mathrm{lpm}$, lo cual indica la existencia de una incompetencia cronotrópica completa. Las pupilas con frecuencia están dilatadas y son pobremente reactivas a la luz. Pueden presentarse varios episodios sincopales diarios y el pronóstico varía desde una recuperación completa hasta un curso debilitante crónico con defectos residuales significativos.

\section{Causas secundarias de disfunción autonómica}

Una variedad amplia de desórdenes puede causar diversos grados de alteración autonómica, algunos de los cuales se resumen en el cuadro 1. Es importante para el médico tener la capacidad de reconocer cuando una disfunción autonómica es parte de una enfermedad sistémica o es una entidad individual. En algunos pacientes pueden coexistir varias condiciones, que llevan a una disminución significativa de la función autonómica.

Cuadro 1. Disfunción autonómica asociada con intolerancia ortostática.

I. Disfunción autonómica primaria
A. Pandisautonomia aguda
B. Falla autonómica pura
C. Atrofia sistémica múltiple
1. Parkinsoniana
2. Piramidal/cerebelar
3. Mixta
D. Síncope reflejo
1. Síncope neurocardiogénico
2. Hipersensibilidad del seno carotídeo

II. Disfuncion autonómica secundaria

A. Origen central

1. Cáncer cerebral

2. Esclerosis múltiple

3. Referida a la edad

4. Siringobulbia

B. Formas periféricas

1. Aferente

a. Síndrome de Guillain-Barré

b. Tabes dorsal

c. Síndrome de Holmes-Adie

2. Eferente

a. Diabetes mellitus

b. Deficiencia de factor de crecimiento del nervio

c. Deficiencia de la dopamina-beta-hidroxilasa

3. Aferente/eferente

a. Disautonomia familiar

4. Origen espinal

a. Mielitis transversa

b. Siringomielia

c.Tumores espinales

5. Otras causas
a. Falla renal
b. Síndromes paraneoplásicos
c. Enfermedad vascular autoinmune/colágeno
d. Infección VIH
e. Amiloidosis
f. Enfermedad de Chagas

Modificado de: Grubb BP, Kosinski D. Dysautonomic and reflex syncope syndromes. Cardiol Clin 1997;15:257-68. (57). Reproducido con autorización del autor. 
En la última década se ha identificado un gran número de anormalidades enzimáticas que pueden resultar en disautonomía. Por ejemplo, el síndrome de deficiencia de la dopamina beta-hidroxilasa, condición que es fácilmente manejada mediante terapia de reemplazo; otros síndromes como el factor de crecimiento neural, la monoaminoxidasa, la L-amino decarboxilasa aromática y algunos neuropéptidos sensitivos pueden resultar en falla autonómica e hipotensión.

Ciertas enfermedades sistémicas y difusas como la falla renal, el cáncer o el sida pueden causar hipotensión y síncope; algunos estudios han mostrado asociación entre hipotensión ortostática y la enfermedad de Alzheimer (24). Es importante tener en cuenta que la intolerancia ortostática puede ser iatrogénica y que pueden causarla una gran cantidad de fármacos. Los fármacos que con más frecuencia se asocian con intolerancia ortostática incluyen los vasodilatadores de acción periférica como los inhibidores de la enzima convertidora de angiotensina, el prazosín, la hidralazina y la guanetidina. Recientemente hemos observado un aumento en la frecuencia de síncope disautonómico en pacientes con falla cardiaca congestiva; en este grupo, la combinación de bajo gasto cardiaco con depleción de volumen debido a la terapia con diuréticos y vasodilatadores interfiere con los mecanismos ya mencionados de adaptación al estrés postural.

En los países latinoamericanos, la enfermedad de Chagas (25) se asocia con disautonomía que afecta los reflejos cardiovasculares, debido a una denervación autonómica crónica de intensidad variable que frecuentemente se relaciona con una respuesta deteriorada al estrés ortostático y que se manifiesta con presíncope o síncope (26). Así mismo, predispone a arritmias malignas incluida la muerte súbita (27). En estudios realizados por nuestro grupo, se ha documentado que la denervación genera una alteración del balance autonómico cardiovascular, caracterizada inicialmente por un incremento relativo del tono simpático y con una disminución del tono vagal, la cual es progresiva y debida a un proceso crónico de denervación y pérdida de los ganglios cardíacos (28-32). Además, en poblaciones susceptibles (países del cono suramericano), la enfermedad puede causar dificultad para la deglución y desórdenes en el movimiento intestinal y alteraciones estructurales (megacolon), relacionadas con alteraciones en los ganglios del plexo mesentérico, causadas por el Trypanosoma cruzi.

\section{Características clínicas}

La característica principal de todas estas alteraciones es la aparición de hipotensión postural. Usualmente, la hipotensión ortostática se define como una caída de la presión arterial sistólica de $20 \mathrm{~mm} \mathrm{Hg}$ durante un período de tres minutos después de asumir la posición erecta. Sin embargo, una caída menor de la presión arterial sistólica, que se asocia con síntomas de ortostatismo, se debe considerar como clínicamente significativa. Un gran porcentaje de estos pacientes presenta una caída lenta y sostenida de la presión arterial en un período de tiempo prolongado (entre 10 y 15 minutos), situación que puede ser muy sintomática. Adicionalmente, la pérdida de la conciencia es lenta y gradual, usualmente al estar caminando o luego de asumir la posición erecta por un tiempo prolongado. Es importante reconocer que con frecuencia los pacientes geriátricos no perciben la reducción de la presión arterial y, por tanto, refieren pocos o ningún síntoma prodrómico previo al episodio sincopal, describiendo estos episodios como un 'ataque con caídas'. En la contraparte están los que presentan pródromos previos como mareo, visión borrosa, visión de estrellas y visión en túnel. Una característica que diferencia el síncope neurocardiogénico y el disautonómico, es que en este último durante los episodios la bradicardia y la diaforesis son poco comunes y, generalmente, se presentan en las horas tempranas de la mañana poco después de asumir la posición erecta. Cualquier factor que lleve a un incremento de la capacitancia venosa periférica tal como el calor extremo, fatiga o la ingestión de alcohol tienden a exacerbar la hipotensión. A medida que evoluciona la enfermedad, algunos pacientes desarrollan una frecuencia cardiaca fija, con mínima respuesta a los cambio posturales o al ejercicio. Adicionalmente, algunos pacientes desarrollan un síndrome de hipertensión supina que alterna con hipotensión postural, 
presumiblemente alteración en la regulación de la vasodilatación en la posición prona. Estos pacientes son un reto terapéutico y son de difícil manejo.

\section{Evaluación de pacientes}

La clave de una adecuada evaluación es la historia clínica y el examen físico minucioso. Las condiciones que precipitan o se asocian con los episodios de síncope o presíncope, cómo se inician, la frecuencia, el patrón y la descripción de los episodios por parte de testigos, son información esencial para la evaluación. Cuando existen otros síntomas asociados aparte del síncope, se debe identificar cuál limita más al paciente. Una historia clínica detallada, con un adecuado examen cardiovascular y neurológico, tiene un gran alcance diagnóstico, superando la solicitud indiscriminada de exámenes múltiples. Los exámenes diagnósticos se deben solicitar de manera dirigida y determinada por los hallazgos de la historia y del examen.

Una revisión extensa de todas las pruebas diagnósticas de evaluación de cada uno de los desordenes autonómicos no es el objetivo de ésta revisión. Para el lector interesado se recomienda la revisión de textos especializados [33-37].

Es importante identificar siempre cualquier fármaco que el sujeto esté tomando, incluso las drogas no alopáticas. Igualmente, cuando un sujeto joven se presenta con síntomas de disfunción autonómica dentro de los diagnósticos diferenciales debe considerarse la posibilidad de abuso de drogas ilícitas o de alcohol. En las mujeres, los síntomas pueden variar de acuerdo con el ciclo menstrual o con el inicio de la menopausia momento en el cual puede haber una leve tendencia hacia la disfunción autonómica.

Dado que las áreas autonómicas del cerebro no son accesibles a la medición directa, se deben utilizar las respuestas de varios órganos a cambios fisiológicos y farmacológicos. Recientemente, se han logrado evaluar los niveles de algunos neurotransmisores y de algunos neuromoduladores autonómicos en líquido cefalorraquídeo y en orina . Dentro estas pruebas, las iniciales son la medición de presión arterial y la respuesta de la frecuencia cardiaca a cambios posicionales, con determinaciones en las posiciones supina, sentado y de pie. El cambio exacto en la presión que puede ser considerado significativo aún está en discusión; sin embargo, usualmente se toma una caída en la presión sistólica entre 20 a 30 $\mathrm{mm} \mathrm{Hg}$ y de la diastólica de 10 a $15 \mathrm{~mm} \mathrm{Hg}$. Es importante recordar el siguiente detalle técnico, cuando se toma la presión al estar de pie la determinación debe ser hecha con el brazo extendido horizontalmente (con el fin de evitar las alteraciones hidrostáticas). Debido a que las respuestas que se obtienen al ponerse de pie son diferentes de las obtenidas pasivamente mediante la inclinación con la mesa basculante, frecuentemente analizamos tanto la respuesta pasiva como activa al reto ortostático. Mayores detalles acerca de esta metodología ha sido descrita en publicaciones previas $[35,36]$. Otras pruebas de evaluación autonómica están también disponibles y tiene gran utilidad en pacientes seleccionados $[36,38,39]$.

\section{Enfoque terapéutico}

Una revisión completa y detallada del manejo terapéutico de los desórdenes autonómicos descritos está fuera del alcance de esta revisión. Para el médico tratante es importante identificar los siguientes aspectos: en cuanto a la naturaleza un síncope hipotensivo puede ser primario o secundario y determinar si existen causas potencialmente reversibles, por ejemplo, drogas, anemia, depleción de volumen, etc.

Es igualmente importante educar al paciente y su familia acerca de las características del problema. La educación debe incluir información para que el paciente evite los factores precipitantes tales como el calor extremo, la deshidratación y el consumo de alcohol, así como lograr que reconozca cualquier síntoma prodrómico y asumir la posición supina al primer indicio de intolerancia ortostática.

El manejo no farmacológico debe utilizarse como primera medida y es de gran utilidad el dormir con la cabecera de la cama elevada (entre 15 y 30 $\mathrm{cm}$ ) y el uso de medias con soporte elástico (presión controlada de 30 a $40 \mathrm{~mm} \mathrm{Hg}$ ). Las técnicas de retroalimentación biológica han demostrado ser útiles en algunas personas así 
Cuadro 2. Opciones terapéuticas.

\begin{tabular}{|c|c|c|}
\hline Terapia & Método o dosis & Problemas comunes \\
\hline Elevación de la cama & Elevación de $45^{\circ}$ de la cama & $\begin{array}{l}\text { Hipotensión, caerse de la cama, } \\
\text { calambres }\end{array}$ \\
\hline $\begin{array}{l}\text { Re-entrenamiento } \\
\text { barorreceptores arteriales }\end{array}$ & $\begin{array}{l}\text { Mesa basculante a } 60^{\circ}, 15 \text { a } 20 \text { minutos } 2 \text { veces } \\
\text { diarias o pararse contra una pared durante } 20 \\
\text { minutos con los pies separados de la pared } \\
30 \text { centímetros. }\end{array}$ & $\begin{array}{l}\text { Difícil implementación y pobre } \\
\text { cumplimiento del tratamiento } \\
\text { crónico }\end{array}$ \\
\hline Soporte elástico & $\begin{array}{l}\text { Requiere media de presión en tobillo al menos de } \\
30-40 \mathrm{~mm} \mathrm{Hg} \text {, mejor si talla fuerte. }\end{array}$ & $\begin{array}{l}\text { Incomodidad, calor, dificultad para } \\
\text { levantarse }\end{array}$ \\
\hline Dieta & $\begin{array}{l}\text { Ingestión de líquidos } 2 \text { a 2,5 L/día } \\
\text { Ingestión de sodio } 150 \text { a } 250 \mathrm{mEq} / \text { día }\end{array}$ & $\begin{array}{l}\text { Hipertensión supina, edema } \\
\text { periférico }\end{array}$ \\
\hline Ejercicio & $\begin{array}{l}\text { Ejercicio aeróbico leve puede ayudar al retorno } \\
\text { venoso. El ejercicio en agua puede ayudar. }\end{array}$ & $\begin{array}{l}\text { Puede bajarse la presión arterial } \\
\text { ante ejercicio vigoroso }\end{array}$ \\
\hline Fluorocortisona & $\begin{array}{l}\text { Iniciar a 0,1-0,2 mg/día, no exceder dosis de } \\
1 \mathrm{mg} / \text { día }\end{array}$ & $\begin{array}{l}\text { Hipopotasemia, hipomagnesemia, } \\
\text { edema periférico, ganancia de } \\
\text { peso, insuficiencia cardíaca } \\
\text { congestiva }\end{array}$ \\
\hline Metilfenidato & $\begin{array}{l}\text { Dar } 5-10 \text { mg por vía oral dos veces al día con las } \\
\text { comidas; dar la última dosis antes de las 6:00 p.m. }\end{array}$ & Náusea, hipertensión supina \\
\hline Midrodrina & $\begin{array}{l}\text { Dar 2,5-10 mg cada } 2-4 \text { horas. Puede usarse hasta } \\
40 \text { mg/día }\end{array}$ & Náusea, hipertensión supina \\
\hline Clonidina & $\begin{array}{l}0,1-0,3 \mathrm{mg} \text { vía oral dos veces al día o parches } \\
\text { semanales }\end{array}$ & $\begin{array}{l}\text { Sequedad de boca, bradicardia, } \\
\text { hipotensión }\end{array}$ \\
\hline Yohimbina & Dar 8 mg por vía oral, dos a tres veces al día & Diarrea, ansiedad, nerviosismo \\
\hline Sulfato de efedrina & Dar 12,5-25 mg por vía oral, tres veces al día & $\begin{array}{l}\text { Taquicardia, temblor, hipertensión } \\
\text { supina }\end{array}$ \\
\hline Fluoxetina & $\begin{array}{l}\text { Dar } 10-20 \text { mg por vía oral, cuatro veces al día } \\
\text { (requiere cuatro semanas de terapia) }\end{array}$ & Náusea, anorexia, diarrea \\
\hline Paroxetina & $\begin{array}{l}\text { Dar } 20-40 \mathrm{mg} \text { por vía oral, dosis única (requiere } \\
\text { cuatro semanas de terapia) }\end{array}$ & $\begin{array}{l}\text { Náusea, somnolencia, diaforesis, } \\
\text { temblor, astenia }\end{array}$ \\
\hline Eritropoyetina & $\begin{array}{l}\text { Aplicar } 4.000 \text { unidades subcutáneas dos veces } \\
\text { a la semana }\end{array}$ & $\begin{array}{l}\text { Ardor en el sitio de inyección, } \\
\text { incremento del hematocrito }\end{array}$ \\
\hline Pindolol & Dar 2,5-5 mg por vía oral, dos o tres veces al día & $\begin{array}{l}\text { Hipotensión, insuficiencia cardíaca } \\
\text { congestiva, bradicardia }\end{array}$ \\
\hline Desmopresina & $\begin{array}{l}\text { Dar 2,5-5 mg por vía oral, dos o tres veces al día. } \\
\text { (análogo de la vasopresina usado como spray nasal) }\end{array}$ & Hiponatremia \\
\hline
\end{tabular}

Modificado de: Grubb BP, Kosinski D. Dysautonomic and reflex syncope syndromes. Cardiol Clin 1997;15:257-68. (57) Reproducido con autorización del autor.

como el reentrenamiento de los barorreceptores arteriales utilizando la mesa basculante han sido de utilidad (cuadro 2).

La terapia farmacológica debe ser usada cuidadosamente y se debe ajustar a las necesidades de acuerdo con el tipo de desorden autonómico, así como los síntomas y condiciones coexistentes (cuadro 2). Debe tenerse siempre en cuenta, que algunos medicamentos pueden potencialmente empeorar los síntomas (efecto prosincopal). En pacientes con síncope neurocardiogénico, varias publicaciones han demostrado que la terapia con betabloqueadores es efectiva [40-42], el efecto es probablemente debido a los efectos inotrópicos negativos que disminuyen la activación de los mecano-rreceptores cardiacos asociado con la caída abrupta del retorno venoso. El incremento en la resistencia vascular periférica que acompaña sin oposición el uso del betabloqueador podría contribuir con sus efectos terapéuticos. No se encuentra utilidad de estos 
fármacos en otras formas de síncope, dado que puede empeorar otros síndromes disautonómicos.

Un agente de gran utilidad en sujetos con síncope disautonómico y en jóvenes con síncope neurocardiogénico es el mineralocorticoide fluorocortisona, sus resultados se explican no sólo por la retención de sodio y fluidos, sino aparentemente por el aumento de la presión a través de un efecto vasoconstrictor indirecto resultante de la sensibilización de los receptores alfa. Debido a que la droga puede ocasionar hipopotasemia e hipomagnesemia, estos dos electrolitos se deben controlar periódicamente.

Debido a la falla existente en la vasoconstricción de los vasos periféricos observada en esta patología, el uso de sustancias vasoconstrictoras ha sido recomendado. Inicialmente se utilizaron sustancias análogas a las anfetaminas como el metilfenidato con excelentes resultados [43]; sin embargo, dado que es una sustancia controlada, con acción potencialmente estimulante a nivel del sistema nervioso central, su uso es limitado. Una excelente alternativa es un agente alfa estimulante, como la midrodrina, el cual no tiene casi efectos sobre el sistema nervioso central o de estimulación cardiaca, mientras que provee iguales grados de estimulación de los receptores alfa periféricos. Varios estudios han demostrado la eficacia de la midrodrina en los desórdenes neurocardiogénicos y en los disautonómicos $[44,45]$.

Se ha encontrado que los agentes bloqueadores de los receptores alfa-2, como la clonidina pueden elevar la presión arterial en pacientes disautonómicos, donde la hipotensión es secundaria a una severa lesión postganglionar simpática [46]. En pacientes con falla autonómica severa, los receptores vasculares alfa-2 postsinápticos (abundantes en el sistema venoso) son hipersensibles. Sin embargo, en sujetos normales la clonidina actúa sobre el sistema nervioso central disminuyendo la descarga simpática y con ello la presión arterial; mientras que en la falla autonómica algunos pacientes exhiben poca o ninguna eferencia simpática, lo cual permite que las acciones periféricas se manifiesten.
Un hallazgo interesante en un buen número de pacientes con falla autonómica es la presencia de anemia. En un estudio de Hoeldtke y Streeten se demostró que las inyecciones subcutáneas de eritropoyetina, además de elevar el conteo celular, incrementaban dramáticamente la presión arterial $[47,48]$; este efecto parece ser independiente del efecto de las células rojas. Un grupo de estudios en modelos animales y humanos han demostrado que el neurotransmisor serotonina (5-hidroxitriptamina) juega un papel esencial en la regulación central de la presión arterial y la frecuencia cardiaca. Ha sido postulado que algunos pacientes con desórdenes autonómicos pudieran tener alteraciones en la producción o regulación de la serotonina central [49-54]. Con base en lo anterior, se ha observado que los inhibidores de la recaptación de la serotonina pueden ser efectivos en el tratamiento del síncope neurocardiogénico y de la hipotensión ortostática.

En pacientes con síncope neurocardiogénico de tipo cardioinhibitorio con frecuentes episodios sincopales, además de riesgo de trauma grave, se ha contemplado el uso de marcapasos bicamerales definitivos. Esta indicación permanece controvertida y existe un potencial efecto placebo importante con el uso de esta terapia $[55,56]$. Debe recordarse, que en los desórdenes disautonómicos, en contraste con el síncope reflejo, el síncope hipotensivo es un único aspecto de una amplia constelación de síntomas relativos a la falla autonómica. El médico no debe dar falsas expectativas sobre cuáles síntomas pueden o no ser eliminados. Tanto el médico como el paciente deben ser conscientes que, por su historia natural, estas patologías son progresivas y que las terapias son más de tratamiento sintomático que etiológico.

\section{Conclusión}

Los desórdenes del control autonómico asociado con intolerancia ortostática son un grupo diverso de enfermedades que pueden resultar en síncope o presíncope. Un entendimiento básico de estos desórdenes es esencial para el diagnóstico y el tratamiento. Los estudios clínicos actualmente en curso ayudarán a definir el espectro de estas alteraciones y a elaborar mejores modalidades diagnósticas y terapéuticas. 


\section{Agradecimiento}

Este artículo esta dedicado a Paul B. Grubb (19241998), padre, guía, amigo y fuente de estímulo.

\section{Referencias}

1. Benarroch E. The central autonomic network: functional organization, dysfunction and perspective. Mayo Clinic Proc 1993;68:988-1001.

2. Ganong WF. Sistema Nervioso Autónomo. En: Ganong WF, editor. Fisiología médica. Décimoquinta edición. México, D.F.: Editorial El Manual Moderno, S.A de C.V; 1996. p.249-56.

3. Vesga BE, Sanabria CL, Leon FE. Regulación autonómica de la frecuencia cardíaca y la presión arterial. Acta Neurol Colomb 2000;16:112-20.

4. Wieling $\mathbf{W}$, Lieshout J. Maintenance of postural normotension in humans. En: Low PA, editor. Clinical autonomic disorders. Second edition. Philadelphia: Lippincott-Raven Publishers; 1997. p.73-82.

5. Joyner MJ, Sheperd JT. Autonomic control of the circulation. En: Low P, editor. Clinical autonomic disorders. Boston: Little Brown; 1993. p.55-67.

6. Grubb BP. Neurocardiogenic syncope. En: Grubb BP, Olshansky B, editors. Syncope: mechanisms y management. Armonk: Futura Publishing; 1998. p.73-106.

7. Kosinski D, Grubb BP, Temesy-Armos P. Pathophysiological aspects of neurocardiogenic syncope. PACE 1995;18:716-21.

8. American Autonomic Society. Consensus statement on the definition of orthostatic hypotension, pure autonomic failure, and multiple system atrophy. Neurology 1996;46:170.

9. Morillo CA, Eckberg DL, Ellenbogen KA, et al. Vagal and sympathetic mechanisms in patients with orthostatic vasovagal syncope. Circulation 1997;96:2509-13.

10. Mosqueda-Garcia R, Furlan R, Fernández-Violante $\mathbf{R}$, et al. Sympathetic and baroreceptor reflex function in neurally mediated syncope evoked by tilt. J Clin Invest 1997;99;2736-44.

11. Vesga BE, Guzmán JC, Lindarte MA, Silva FA, Morillo CA. Utilidad de la mesa basculante y las pruebas autonómicas en 934 sujetos con sospecha de síncope. Acta Méd Colomb 2002;27:324.

12. Sutton $\mathbf{R}$, Petersen $\mathbf{M}$. The clinical spectrum of neurocardiogenic syncope. J Cardiovasc Electrophysiol 1995;6:569-76.

13. Morillo CA, Camacho ME, Wood MA, Gilligan DM, Ellenbogen KA. Diagnostic utility of mechanical, pharmacological, and orthostatic stimulation of the carotid sinus in patients with unexplained syncope. J Am Coll Cardiol 1999;34:1587-94.
14. Kosinski D. Miscellaneous causes of syncope. En: Grubb BP, Olshansky, editors. Syncope: mechanisms y management. Armonk: Futura Publishing; 1998. p.297304.

15. Bradbury S, Eggleston C. Postural hypotension: a report of three cases. Am Heart J 1925;1:73-86.

16. Robetson D, Polinsky R. A primer on the autonomic nervous system. San Diego: Academic Press; 1996.

17. Shy GM, Drager GA. A neurologic syndrome associated with orthostatic hypotension. Arch Neurol 1960;3: 511-27.

18. Mathias CJ. The classification and nomenclature of autonomic disorders: ending chaos, restoring conflict, and hopefully achieving clarity. Clin Auton Res 1995;5: 307-10.

19. Grubb BP, Kosinski D, Boehm K, Kip K. The postural orthostatic tachycardia syndrome: a neurocardiogenic variant identified during head up tilt table testing. PACE 1997;20:2205-12.

20. Morillo CA, Klein GJ, Thakur RK, Li HG, Yee R. Mechanism of inappropriate sinus tachycardia: role of sympathovagal balance. Circulation 1994;90:873-7.

21. Bou-Holaig I, Rowe P, Kan J, Calkins H. The relationship between neurally mediated hypotension and chronic fatigue syndrome. JAMA 1995;274:961-7.

22. Schondorf R, Freeman R. The importance of orthostatic intolerance in the chronic fatigue syndrome. Am J Med Sci 1999;317:117-23.

23. Grubb BP, Kosinski D. Acute pandysautonomic syncope. Eur J Cardiac Pacing and Electrophysiol 1997;7: 10-4.

24. Passant V, Warkentin S, Karlson, et al. Orthostatic hypotension in organic dementia: relatioship between blood pressure, cortical blood flow, and symptoms. Clin Auton Res 1996;6:29-36.

25. losa D, Dequattro V, De Ping-Lee D. Pathogenesis of cardiac neuromyopathy in Chagas' disease and the role of the autonomic nervous system. J Auton Syst 1990;30:S83-8.

26. Lazzari JO. Autonomic nervous system alterations in Chagas' disease: review of the literature. En: Chagas' disease and the nervous system. Washington, D.C.: Panamerican Health Organization; 1994. p.72-95.

27. Diaz JO, Makikallio TH, Huikuri HV, et al. Heart rate dynamics before the spontaneous onset of ventricular tachyarrhythmias in Chagas' heart disease. Am J Cardiol 2001;87:1123-5.

28. Belloti G, Bocci EA, Moraes AV et al. In vivo detection of Trypanosoma cruzi antigens in hearts of patients with chronic Chagas' disease. Am Heart J 1996; 131:301-7. 
29. Rossi M. Patogênese da miocardite chagásica crônica. Rev Soc Cardiol Estado de Sâo Paulo 1994;2:85-9.

30. Morillo CA. Effects of Chagas' disease on cardiac autonomic reflex function. En: Tentory MC, Segura EL, Hayes DL, editors. Arrhythmia management in Chagas' disease. Armonk, NY: Futura Publishing Co., Inc; 2000. p.51-65.

31. Villar JC, Vega A, León H, Contreras JP, Amado PM, Pradilla LP, Morillo CA. Cambios en la función autonómica cardiaca en sujetos seropositivos a T. cruzi asintomáticos. Rev Col Cardiol 1997;14:43-9.

32. Morillo CA, Schultz R, López-Jaramillo $P$, et al. Predicting progression to chagasic cardiomiopathy: from cellular mechanism to diagnostic approaches. Role of inflammatory, genetic and autonomic markers. Cardiol al día 2000;3:83-5.

33. Bannister R, Mathias C. Autonomic failure: a textbook of clinical disorders of the autonomic nervous system. Oxford: Oxford Medical Publications; 1992.

34. Low PA, editor. Clinical autonomic disorders. Second edition. Philadelphia: Lippincott-Raven Publishers; 1997.

35. Grubb BP, Olshansky B, editors. Syncope: mechanisms y management. Armonk, NY: Futura Publishing; 1998.

36. Chaves AM, Vesga BE, Rueda OL, et al. Pruebas para la evaluación del sistema nervioso autónomo. Salud UIS 1998;28:62-70.

37. Robertson D, Biaggioni I. Disorders of the autonomic nervous system. London: Harwood Academic Publishers; 1995.

38. Grubb BP, Kosinski D. Tilt table test: concepts and limitations. PACE 1997;20:781-7.

39. Kapoor WN. Using a tilt table test to evaluate syncope. Am J Med Sci 1999;317:110-6.

40. Cox MM, Perlman BA, Mayor MR, et al. Acute and long-term beta-adrenergic blockade for patients with neurocardiogenic syncope. J Am Coll Cardiol 1995;26: 1293-8.

41. Sheldon RS, Rose S, Flanagan P, Koshman ML, Killan S. Effect of beta blockers on the time to first syncope recurrence in patients after a positive isoproterenol-tilt table test. Am J Cardiol 1996;78:536-9.

42. Natale A, Newby KH, Dhala A, Akhtar M, Sra J. Response to beta blockers in patients with neurocardiogenic syncope: how to predict beneficial effects. J Cardiovasc Electrophysiol 1996;7:1154-8.

43. Grubb BP, Kosinski D, Mouhaffel A, Pothoulaskis A. The use of methylphenidate in the treatment of refratory neurocardiogenic syncope. PACE 1996;19: $836-40$
44. Low P, Gilden J, Freeman R. Efficacy of midodrine versus placebo in neurocardiogenic orthostatic hypotension. JAMA 1997;277:1046-51.

45. Sra J, Maglio C, BiehI M. Efficacy of midrodrine hydrochloride in neurocardiogenic syncope refractory to standard terapy. J Cardiovasc Electrophysiol 1997; 8:42-6.

46. Robertson D, Davis TL. Recent advances in the treatment of orthostatic hypotension. Neurology 1995;5:526-32.

47. Hoeldtke RD, Streeton DH. Treatment of orthostatic hypotension with erythropoietin. N Engl J Med 1993;329: 611-5.

48. Grubb BP, Lachant N, Kosinski D. Erythropoietin as a therapy for severe refractory orthostaic hypotension. Clin Auton Res 1994;4:212.

49. Grubb BP, Kosinski D. Serotonin and syncope: an emerging connection? Eur J Cardiac Pacing and Electrophysiol 1996;5:306-14.

50. Grubb BP, Samoil D, Kosinski D. Fluoxetine hydrochloride for the treatment of severe refractory orthostatic hypotension. PACE 1993;16:801-5.

51. Guzmán JC, Lindarte MA, Morillo CA. Síncope neurocardiogénico: alteración del sistema central serotoninérgico corticotrópico. Cardiol al día 2001; 4:81-5.

52. Guzmán JC, Vesga BE, Silva FA, Lindarte MA, Morillo CA. Síncope neurocardiogénico y trastornos del estado de ánimo: ¿una vía común mediada por el eje serotoninérgico central? Acta Méd Colomb 2002; $27: 323$.

53. Ruggiero DA, Underwood MD, Rice PM, Mann JJ, Arango V. Corticotropic-releasing hormone and serotonin interact in the human brainstem: behavioral implications, Neuroscience 1999;91:1343-54.

54. Knowles ID, Ramage AG. Evidence for a role for central 5-HT2B as well as 5-HT2A receptors in cardiovascular regulation in anaesthetized rats. $\mathrm{Br} J$ Pharmacol 1999;128:530-42.

55. Benditt D, Petersen ME, Lurie K. Cardiac pacing for prevention of recurrent vasovagal syncope. Ann Int Med 1995;122:204-9.

56. Connolly S, Sheldon R, Roberts R, Gent M, on behalf of the VPS investigators. The North American vasovagal pacemaker study (VPS). A randomized trial of permanent cardiac pacing for the prevention of vasovagal syncope. J Am Coll Cardiol 1999;33:1620 .

57. Grubb BP, Kosinsky D. Dysautonomic and reflex syncope syndromes. Cardiol Clin 1997;15:257-68. 